\title{
Veterinary Research reviewer acknowledgement 2015
}

Michel Brémont ${ }^{*}$ and Élodie Coulamy

The Veterinary Research editorial team would sincerely like to thank all of our reviewers who contributed to peer review for the journal in 2015.

\begin{tabular}{|c|c|c|}
\hline $\begin{array}{l}\text { Mohamed Abdul-Careem } \\
\text { Canada }\end{array}$ & $\begin{array}{l}\text { Walid Azab } \\
\text { Germany }\end{array}$ & $\begin{array}{l}\text { Sven M. Bergmann } \\
\text { Germany }\end{array}$ \\
\hline $\begin{array}{l}\text { Cristina Acin } \\
\text { Spain }\end{array}$ & $\begin{array}{l}\text { V. Balamurugan } \\
\text { India }\end{array}$ & $\begin{array}{l}\text { Paula Berguer } \\
\text { Antigua and Barbuda }\end{array}$ \\
\hline $\begin{array}{l}\text { Pier Luigi Acutis } \\
\text { Italy }\end{array}$ & $\begin{array}{l}\text { Mitchell Balish } \\
\text { USA }\end{array}$ & $\begin{array}{l}\text { Jean-Francois Bernardet } \\
\text { France }\end{array}$ \\
\hline $\begin{array}{l}\text { Ben Adler } \\
\text { Australia }\end{array}$ & $\begin{array}{l}\text { Keith Ballingall } \\
\text { UK }\end{array}$ & $\begin{array}{l}\text { Helle Bielefeldt-Ohmann } \\
\text { Australia }\end{array}$ \\
\hline $\begin{array}{l}\text { Judd Aiken } \\
\text { Canada }\end{array}$ & $\begin{array}{l}\text { Isabel Bandin } \\
\text { Spain }\end{array}$ & $\begin{array}{l}\text { Charalambos Billinis } \\
\text { Greece }\end{array}$ \\
\hline $\begin{array}{l}\text { Raúl Almeida } \\
\text { USA }\end{array}$ & $\begin{array}{l}\text { Ashley Banyard } \\
\text { UK }\end{array}$ & $\begin{array}{l}\text { Pat Blackall } \\
\text { Australia }\end{array}$ \\
\hline $\begin{array}{l}\text { Rodrigo Almeida-Paes } \\
\text { Brazil }\end{array}$ & $\begin{array}{l}\text { Paul Bartley } \\
\text { UK }\end{array}$ & $\begin{array}{l}\text { Barbara Blacklaws } \\
\text { UK }\end{array}$ \\
\hline $\begin{array}{l}\text { Irina Amorim } \\
\text { Portugal }\end{array}$ & $\begin{array}{l}\text { Christoph Baums } \\
\text { Germany }\end{array}$ & $\begin{array}{l}\text { Damer Blake } \\
\text { UK }\end{array}$ \\
\hline $\begin{array}{l}\text { Thavamathi Annamalai } \\
\text { USA }\end{array}$ & $\begin{array}{l}\text { Brad Bearson } \\
\text { USA }\end{array}$ & $\begin{array}{l}\text { Sandra Blome } \\
\text { Germany }\end{array}$ \\
\hline $\begin{array}{l}\text { Virginia Aragon } \\
\text { Spain }\end{array}$ & $\begin{array}{l}\text { Karen Beemon } \\
\text { USA }\end{array}$ & $\begin{array}{l}\text { Anders Bojesen } \\
\text { Denmark }\end{array}$ \\
\hline $\begin{array}{l}\text { Cova Arias } \\
\text { USA }\end{array}$ & $\begin{array}{l}\text { Martin Beer } \\
\text { Germany }\end{array}$ & $\begin{array}{l}\text { Manuel Vitor Borca } \\
\text { USA }\end{array}$ \\
\hline $\begin{array}{l}\text { Brian Austin } \\
\text { UK }\end{array}$ & $\begin{array}{l}\text { Douglas Begg } \\
\text { Australia }\end{array}$ & $\begin{array}{l}\text { Berend-Jan Bosch } \\
\text { The Netherlands }\end{array}$ \\
\hline $\begin{array}{l}\text { María Auxiliadora Dea-Ayuela } \\
\text { Spain }\end{array}$ & $\begin{array}{l}\text { David Benfield } \\
\text { USA }\end{array}$ & $\begin{array}{l}\text { Paolo Bosi } \\
\text { Italy }\end{array}$ \\
\hline
\end{tabular}

(C) 2016 Brémont and Coulamy. This article is distributed under the terms of the Creative Commons Attribution 4.0 International 


\begin{tabular}{|c|c|c|}
\hline Cristina Botías & Ulas Cinar & Nicholas Davis-Poynter \\
\hline UK & USA & Australia \\
\hline $\begin{array}{l}\text { John Boyce } \\
\text { USA }\end{array}$ & $\begin{array}{l}\text { Edwin Claerebout } \\
\text { Belgium }\end{array}$ & $\begin{array}{l}\text { Jeroen De Buck } \\
\text { Canada }\end{array}$ \\
\hline $\begin{array}{l}\text { Filip Boyen } \\
\text { Belgium }\end{array}$ & $\begin{array}{l}\text { Tracy Clegg } \\
\text { Ireland }\end{array}$ & $\begin{array}{l}\text { Eva De Clercq } \\
\text { Belgium }\end{array}$ \\
\hline $\begin{array}{l}\text { Angele Breithaupt } \\
\text { Germany }\end{array}$ & $\begin{array}{l}\text { Lee Cohnstaedt } \\
\text { USA }\end{array}$ & $\begin{array}{l}\text { Astrid De Greeff } \\
\text { The Netherlands }\end{array}$ \\
\hline $\begin{array}{l}\text { Michel Brémont } \\
\text { France }\end{array}$ & $\begin{array}{l}\text { Esther Collantes Fernandez } \\
\text { Spain }\end{array}$ & $\begin{array}{l}\text { Henri De Greve } \\
\text { Belgium }\end{array}$ \\
\hline $\begin{array}{l}\text { Robert Briggs } \\
\text { USA }\end{array}$ & $\begin{array}{l}\text { Ellen Collisson } \\
\text { USA }\end{array}$ & $\begin{array}{l}\text { Teresa De Los Santos } \\
\text { USA }\end{array}$ \\
\hline $\begin{array}{l}\text { Collette Britton } \\
\text { UK }\end{array}$ & $\begin{array}{l}\text { Andrew Conlan } \\
\text { UK }\end{array}$ & $\begin{array}{l}\text { Annemie Decostere } \\
\text { Belgium }\end{array}$ \\
\hline $\begin{array}{l}\text { Susan Brockmeier } \\
\text { USA }\end{array}$ & $\begin{array}{l}\text { Tim Connelley } \\
\text { UK }\end{array}$ & $\begin{array}{l}\text { Aldo Dekker } \\
\text { The Netherlands }\end{array}$ \\
\hline $\begin{array}{l}\text { Elisabetta Buommino } \\
\text { Italy }\end{array}$ & $\begin{array}{l}\text { Christopher Connolly } \\
\text { UK }\end{array}$ & $\begin{array}{l}\text { Bert Devriendt } \\
\text { Belgium }\end{array}$ \\
\hline $\begin{array}{l}\text { Stewart Burgess } \\
\text { UK }\end{array}$ & $\begin{array}{l}\text { Brian Cooke } \\
\text { Australia }\end{array}$ & $\begin{array}{l}\text { Jeroen Dewulf } \\
\text { Belgium }\end{array}$ \\
\hline $\begin{array}{l}\text { Christian Burvenich } \\
\text { Belgium }\end{array}$ & $\begin{array}{l}\text { Benjamín Costas } \\
\text { Portugal }\end{array}$ & $\begin{array}{l}\text { Barbara Di Martino } \\
\text { Italy }\end{array}$ \\
\hline $\begin{array}{l}\text { Luis Calvinho } \\
\text { Argentina }\end{array}$ & $\begin{array}{l}\text { Paul Coussens } \\
\text { USA }\end{array}$ & $\begin{array}{l}\text { Adama Diallo } \\
\text { Austria }\end{array}$ \\
\hline $\begin{array}{l}\text { Julien Cappelle } \\
\text { France }\end{array}$ & $\begin{array}{l}\text { Eric Cox } \\
\text { Belgium }\end{array}$ & $\begin{array}{l}\text { Colette Dissous } \\
\text { France }\end{array}$ \\
\hline $\begin{array}{l}\text { Lorenzo Capucci } \\
\text { Italy }\end{array}$ & $\begin{array}{l}\text { Alberto Cuesta } \\
\text { Spain }\end{array}$ & $\begin{array}{l}\text { Linda Dixon } \\
\text { UK }\end{array}$ \\
\hline $\begin{array}{l}\text { Librado Carrasco } \\
\text { Spain }\end{array}$ & $\begin{array}{l}\text { John Curran } \\
\text { Australia }\end{array}$ & $\begin{array}{l}\text { Carlos P. Dopazo } \\
\text { Spain }\end{array}$ \\
\hline $\begin{array}{l}\text { Phillip Cassey } \\
\text { Australia }\end{array}$ & $\begin{array}{l}\text { Marcia Dalastra Laurenti } \\
\text { Brazil }\end{array}$ & $\begin{array}{l}\text { Fernanda Doréa } \\
\text { Canada }\end{array}$ \\
\hline $\begin{array}{l}\text { Joaquin Castilla } \\
\text { Spain }\end{array}$ & $\begin{array}{l}\text { Sylvie Daminet } \\
\text { Belgium }\end{array}$ & $\begin{array}{l}\text { Michael Dove } \\
\text { Australia }\end{array}$ \\
\hline $\begin{array}{l}\text { Elena Chaves-Pozo } \\
\text { Spain }\end{array}$ & $\begin{array}{l}\text { Rohana Dassanayake } \\
\text { USA }\end{array}$ & $\begin{array}{l}\text { Charles Martin Dozois } \\
\text { Canada }\end{array}$ \\
\hline $\begin{array}{l}\text { Yahia Chebloune } \\
\text { France }\end{array}$ & $\begin{array}{l}\text { James Daubenspeck } \\
\text { USA }\end{array}$ & $\begin{array}{l}\text { Jitender Dubey } \\
\text { USA }\end{array}$ \\
\hline $\begin{array}{l}\text { Koen Chiers } \\
\text { Belgium }\end{array}$ & $\begin{array}{l}\text { Stephen Davis } \\
\text { Australia }\end{array}$ & $\begin{array}{l}\text { Richard Ducatelle } \\
\text { Belgium }\end{array}$ \\
\hline $\begin{array}{l}\text { Pinwen Chiou } \\
\text { Taiwan, ROC }\end{array}$ & $\begin{array}{l}\text { William Davis } \\
\text { USA }\end{array}$ & $\begin{array}{l}\text { Luis Enjuanes } \\
\text { Spain }\end{array}$ \\
\hline
\end{tabular}




\begin{tabular}{|c|c|c|}
\hline Gary Entrican & Juan J. Garrido & Charles Haley \\
\hline UK & Spain & USA \\
\hline Helena Eriksson & Joseba M. Garrido & Sabine Hammer \\
\hline Sweden & Spain & Austria \\
\hline Cesar Escobedo-Bonilla & M. Carolyn Gates & John Hammond \\
\hline Mexico & UK & UK \\
\hline Roger Evans & Volker Gerdts & Josee Harel \\
\hline UK & Canada & Canada \\
\hline Oystein Evensen & Wilhelm Gerner & David Harper \\
\hline Norway & Austria & UK \\
\hline Pauline Ezanno & Amanda Gibson & Balazs Harrach \\
\hline France & UK & Hungary \\
\hline Kay Faaberg & Elisabetta Giuffra & Jiří Hejnar \\
\hline USA & France & Czech Republic \\
\hline Ten Feizi & Bruno Goddeeris & Andrew Hemphill \\
\hline UK & Belgium & Switzerland \\
\hline Giancarlo Ferrari & William Golde & Tharangani Herath \\
\hline Italy & USA & UK \\
\hline Jaime Figueroa & Ernest A. Gould & Katleen Hermans \\
\hline Chile & UK & Belgium \\
\hline Katja Fischer & Luigi Gradoni & Georg Herrler \\
\hline Australia & Italy & Germany \\
\hline U. Fischer & David Graham & Michael Hess \\
\hline Germany & UK & Austria \\
\hline Bram Flahou & Simon Graham & Myriam Hesta \\
\hline Belgium & UK & Belgium \\
\hline Caroline Frey & Daniel Grenier & Douglas Hooper \\
\hline Switzerland & Canada & USA \\
\hline Hideto Fukushi & Andrea Gröne & John Hopkins \\
\hline Japan & The Netherlands & UK \\
\hline Sarah Gabriël & Martin H. Groschup & Tiago Hori \\
\hline Belgium & Germany & Canada \\
\hline Nellie Gagne & Jean Luc Guérin & Margaret Hosie \\
\hline Canada & France & UK \\
\hline Carmina Gallardo & Yusong Guo & Kurt Houf \\
\hline Spain & USA & Belgium \\
\hline Cris Gallardo-Escárate & Efrain Guzman & Charlie Hsu \\
\hline Chile & UK & USA \\
\hline Yulong Gao & Freddy Haesebrouck & Falk Huettmann \\
\hline China & Belgium & USA \\
\hline José A. García-Cabrera & Katsuro Hagiwara & William Hughes \\
\hline Spain & Japan & UK \\
\hline
\end{tabular}




\begin{tabular}{|c|c|c|}
\hline $\begin{array}{l}\text { Ana Hurtado } \\
\text { Spain }\end{array}$ & $\begin{array}{l}\text { Christoph Koch } \\
\text { Switzerland }\end{array}$ & $\begin{array}{l}\text { Yanmin Li } \\
\text { USA }\end{array}$ \\
\hline $\begin{array}{l}\text { Gisela Hussey } \\
\text { USA }\end{array}$ & $\begin{array}{l}\text { Guus Koch } \\
\text { The Netherlands }\end{array}$ & $\begin{array}{l}\text { Dieter Liebhart } \\
\text { Austria }\end{array}$ \\
\hline $\begin{array}{l}\text { Mike Hutchings } \\
\text { UK }\end{array}$ & $\begin{array}{l}\text { Frank Koenen } \\
\text { Belgium }\end{array}$ & $\begin{array}{l}\text { Hyun Lillehoj } \\
\text { USA }\end{array}$ \\
\hline $\begin{array}{l}\text { Antti Iivanainen } \\
\text { Finland }\end{array}$ & $\begin{array}{l}\text { Wolfgang Koester } \\
\text { Canada }\end{array}$ & $\begin{array}{l}\text { John Lippolis } \\
\text { USA }\end{array}$ \\
\hline $\begin{array}{l}\text { Thomas Inzana } \\
\text { USA }\end{array}$ & $\begin{array}{l}\text { Ad Koets } \\
\text { The Netherlands }\end{array}$ & $\begin{array}{l}\text { Tom Lipscomb } \\
\text { USA }\end{array}$ \\
\hline $\begin{array}{l}\text { Mario Jacques } \\
\text { Canada }\end{array}$ & $\begin{array}{l}\text { Michael Kogut } \\
\text { USA }\end{array}$ & $\begin{array}{l}\text { Annette Litster } \\
\text { USA }\end{array}$ \\
\hline $\begin{array}{l}\text { Mark Jenkins } \\
\text { USA }\end{array}$ & $\begin{array}{l}\text { Nicholas Komar } \\
\text { USA }\end{array}$ & $\begin{array}{l}\text { Ding Xiang Liu } \\
\text { Singapore }\end{array}$ \\
\hline $\begin{array}{l}\text { Kirsty Jensen } \\
\text { UK }\end{array}$ & $\begin{array}{l}\text { Peter Kuhnert } \\
\text { Switzerland }\end{array}$ & $\begin{array}{l}\text { Hung-Jen Liu } \\
\text { Taiwan, ROC }\end{array}$ \\
\hline $\begin{array}{l}\text { Anja Joachim } \\
\text { Austria }\end{array}$ & $\begin{array}{l}\text { Gael Kurath } \\
\text { USA }\end{array}$ & $\begin{array}{l}\text { Remo Lobetti } \\
\text { Zambia }\end{array}$ \\
\hline $\begin{array}{l}\text { Nick Johnson } \\
\text { UK }\end{array}$ & $\begin{array}{l}\text { Michael Kurth } \\
\text { Germany }\end{array}$ & $\begin{array}{l}\text { Willie Loeffen } \\
\text { The Netherlands }\end{array}$ \\
\hline $\begin{array}{l}\text { Clinton Jones } \\
\text { USA }\end{array}$ & $\begin{array}{l}\text { Andrea Ladinig } \\
\text { Austria }\end{array}$ & $\begin{array}{l}\text { Joel López-Meza } \\
\text { Mexico }\end{array}$ \\
\hline $\begin{array}{l}\text { Clint Jones } \\
\text { USA }\end{array}$ & $\begin{array}{l}\text { Jan Langeveld } \\
\text { The Netherlands }\end{array}$ & $\begin{array}{l}\text { Niels Lorenzen } \\
\text { Denmark }\end{array}$ \\
\hline $\begin{array}{l}\text { Kelli Jones } \\
\text { USA }\end{array}$ & $\begin{array}{l}\text { Cristina Lanzas } \\
\text { USA }\end{array}$ & $\begin{array}{l}\text { N. James Maclachlan } \\
\text { USA }\end{array}$ \\
\hline $\begin{array}{l}\text { Ramon A. Juste } \\
\text { Spain }\end{array}$ & $\begin{array}{l}\text { Neus Latorre-Margalef } \\
\text { USA }\end{array}$ & $\begin{array}{l}\text { Lone Madsen } \\
\text { Denmark }\end{array}$ \\
\hline $\begin{array}{l}\text { Shaden Kamhawi } \\
\text { USA }\end{array}$ & $\begin{array}{l}\text { Daniel Layton } \\
\text { Australia }\end{array}$ & $\begin{array}{l}\text { Dominiek Maes } \\
\text { Belgium }\end{array}$ \\
\hline $\begin{array}{l}\text { Bernd Kaspers } \\
\text { Germany }\end{array}$ & $\begin{array}{l}\text { Ghislaine Le Gall-Recule } \\
\text { France }\end{array}$ & $\begin{array}{l}\text { Marc Marenda } \\
\text { Australia }\end{array}$ \\
\hline $\begin{array}{l}\text { Anthony Keyburn } \\
\text { Australia }\end{array}$ & $\begin{array}{l}\text { Ronan Le Goffic } \\
\text { France }\end{array}$ & $\begin{array}{l}\text { Marc Maresca } \\
\text { France }\end{array}$ \\
\hline $\begin{array}{l}\text { Subodh Kishore } \\
\text { India }\end{array}$ & $\begin{array}{l}\text { Jacques Le Pendu } \\
\text { France }\end{array}$ & $\begin{array}{l}\text { Raquel Marques } \\
\text { Portugal }\end{array}$ \\
\hline $\begin{array}{l}\text { Guilherme Klafke } \\
\text { Brazil }\end{array}$ & $\begin{array}{l}\text { Sandrine Lesellier } \\
\text { UK }\end{array}$ & $\begin{array}{l}\text { Paolo Martelli } \\
\text { Italy }\end{array}$ \\
\hline $\begin{array}{l}\text { Doris Klingelhoefer } \\
\text { Germany }\end{array}$ & $\begin{array}{l}\text { Martin Lessard } \\
\text { Canada }\end{array}$ & $\begin{array}{l}\text { Enric Mateu } \\
\text { Spain }\end{array}$ \\
\hline $\begin{array}{l}\text { Don Klinkenberg } \\
\text { The Netherlands }\end{array}$ & $\begin{array}{l}\text { Hong Li } \\
\text { USA }\end{array}$ & $\begin{array}{l}\text { Mikhail Matrosovich } \\
\text { Germany }\end{array}$ \\
\hline
\end{tabular}


Marian Mcloughlin

UK

Tom McNeilly

UK

Kieran Meade

Ireland

Melha Mellata

USA

Xiang-Jin Meng

USA

Francois Meurens

France

John Middleton

USA

Simon Milling

UK

Rebecca Mitchell

USA

Adebayo Molehin

Australia

Maria Montoya

UK

Robert Moore

Australia

Héctor Mora Montes

Mexico

David Moran

Guatemala

Kate Mounsey

Australia

Joachim Mueller

Switzerland

Eann Munro

UK

Alexander Murray

UK

Michael Murtaugh

USA

K. Naeem

Pakistan

Venugopal Nair

UK
Hans Nauwynck

Belgium

Olivier Neyrolles

France

Robin Nicholas

UK

Tokohiko Nishizawa

Republic Of Korea

Pierre Nouvellet

UK

Niels Olesen

Denmark

Guilherme Oliveira

Brazil

Steven Olsen

USA

Luis Miguel Ortega-Mora

Spain

Angel Ortiz Pelaez

Italy

Pamela Osterlund

Finland

Romain Paillot

UK

Richard Paley

UK

Bongkyun Park

Republic of Korea

John Pasick

Canada

Nicole Pavio

France

Luc Peelman

Belgium

Andres Perez

USA

Elisa Pérez-Ramírez

Spain

Patricia Pesavento

USA

Wolfram Petzl

Germany
Maria Piccone

USA

Sofie Piepers

Belgium

Maria Pieters

Spain

Patrick Pithua

USA

A. Poli

Italy

Jamie Prentice

UK

John Prescott

Canada

Cinta Prieto

Spain

Maureen Purcell

USA

Qiwei Qin

China

Valeria Quattrocchi

Argentina

Pascal Rainard

France

Silke Rautenschlein

Germany

Krystle Reagan

USA

Patrick Redig

USA

Gourapura Renukaradhya USA

S. Richart

USA

Espen Rimstad

Norway

Katherine Roberts

UK

Fernando Rodríguez

Spain

Alexander Rodriguez-Palacios USA 
Wendi Roe

New Zealand

Angela Römer-Oberdörfer

Germany

Julian Rood

Australia

Mirko Rossi

Finland

\section{Raymond Rowland}

USA

Charles Rupprecht

USA

Ivan Rychlik

Czech Republic

Mariana Sa E. Silva

USA

Armin Saalmueller

Austria

Yoshihiro Sakoda

Japan

Javier Salguero

UK

Oscar Daniel Salomón

Argentina

Siba Samal

USA

Yongming Sang

USA

Gereon Schares

Germany

Mariela Segura

Canada

Emmanuel Serrano

Spain

Noemi Sevilla

Spain

Hans-Martin Seyfert

Germany

I. Martin Sheldon

UK

Huigang Shen

USA
Susan Shriner

USA

Theodoros Sklaviadis

Greece

Kerstin Skovgaard

Denmark

Jan Šlapeta

Australia

Annemieke Smet

Belgium

Krzysztof Śmietanka

Poland

Hilde Smith

The Netherlands

Ken Smith

UK

Rebecca Smith

USA

Adrian Smith

UK

Daesub Song

North Korea

Maria Rathmann Sørensen

Denmark

Marcelo Soria

Argentina

Esteban Soto

Saint Kitts And Nevis

Sandra Souto

Spain

Simon Spencer

UK

Marina Spinu

Romania

John Spiropoulos

UK

Barry Stein

USA

Sebastian Steinfartz

Germany

Sophie St-Hilaire

Canada
Norbert Stockhofe-Zurwieden

The Netherlands

David Stone

UK

Artur Summerfield

Switzerland

Lotta-Riina Sundberg

Finland

Christina Swaggerty

USA

Dustin Swanson

USA

Raymond Sweeney

USA

Fumihiro Taguchi

Japan

Eileen Thacker

USA

Alexandre Thibodeau

Canada

Suresh Tikoo

Canada

Ian Tizard

USA

Juan Maria Torres

Spain

Sabine Totemeyer

UK

Paolo Trevisi

Italy

Vasilios Tsiouris

Greece

Chris Tuggle

USA

Maria Tunon

Spain

Kevin Tyler

UK

Gabriele Vaccari

Italy

Jean-Francois Valarcher Sweden 
Peter Valentin-Weigand

Germany

Thierry Van Den Berg

Belgium

Filip Van Immerseel

Belgium

Jeroen Van Leuken

The Netherlands

Alain Vanderplasschen

Belgium

Daisy Vanrompay

Belgium

Anouk Veldhuis

The Netherlands

Lonneke Vervelde

UK

Mafalda Viana

UK

Stefan Vilcek

Slovakia

\section{Amy Vincent}

USA

Andrea Vögtlin

Switzerland

Jiri Volf

Czech Republic
Victoriya Volkova

USA

Wilna Vosloo

Australia

Bettina Wagner

USA

Qihong Wang

USA

Hao-Ching Wang

Taiwan, ROC

Lingshu Wang

USA

Zhiliang Wang

China

Karin Weber

Germany

Neil Wedlock

New Zealand

Eefke Weesendorp

The Netherlands

Dirk Werling

UK

Christopher Whitehouse USA

Geert Wiegertjes

The Netherlands
Diana Williams

UK

Jim Winton

USA

Robert Wolf

Canada

Guanghui Wu

UK

Feng Yang

China

Joo Mi Yi

Republic of Korea

Han Sang Yoo

Republic of Korea

Ruth Zadoks

UK

Huanmin Zhang

USA

Yan Zhang

USA

Yanjin Zhang

USA

En-Min Zhou

China

Stephan Zientara

France 